\title{
In-band Spectrum Sensing Method Based on Guard Frequency Bandwidth for Cognitive OFDM Systems
}

\author{
Guoqing Jia ${ }^{1, \mathrm{a}}$, Shanji Chen ${ }^{1}$, Chao Chen ${ }^{1}$, and Xiaohong $\mathrm{Ji}^{1}$ \\ ${ }^{1}$ Qinghai University for Nationalities, No.3 Middle Bayi Road, Xining, China
}

\begin{abstract}
A novel in-band spectrum sensing method based on guard frequency bandwidth for cognitive OFDM systems is proposed in this paper. The proposed spectrum sensing procedure is based on leakagenegligible-BW, on which power detection or other detection methods can be implemented. The advantage of this in-band sensing method is the impact to OFDM system frame structure could be minimized.
\end{abstract}

Keywords: OFDM System; Cognitive Radio; In-band Sensing; Guard Band

\section{Introduction}

As the development of wireless communications, there are fewer and fewer spectrum resources which can be allocated to a new implemented radio transmission system. However, the frequency utilization efficiency is very low for some wireless communication systems, such as TV broadcast system. So, cognitive radio technology[1-5] has been developed to allow unlicensed system (secondary system) to exploit spectrum of licensed system (primary system) opportunistically. Thus the spectrum allocation problem can be solved and the spectrum utilization efficiency could be promoted greatly[6-9].

OFDM (Orthogonal Frequency Division Multiplexing) is accepted as the most promising technique for next generation wireless communication system. The system BW (BandWidth) for wireless OFDM system could be as much as $100 \mathrm{MHz}$, such as LTE-A (Long Term Evolution Advanced) system. In addition, there is further need for spectrum for femtocell implementation, etc. So the spectrum allocation for wireless OFDM system will be very difficult as no more available wide band spectrum can be used. As a result, it is very important for wireless OFDM system to sense and reuse the frequency resource of licensed systems which has low spectrum utilization efficiency. Figure 1 gives an illustration of wireless communication system existing in TV spectrum.

In figure 1 , the procedure of spectrum sensing by wireless communication system is composed of two scenarios:

- Out-band frequency spectrum sensing: the procedure of sensing a free spectrum band of primary system by secondary system;
-In-band frequency spectrum sensing: the procedure of sensing to detect whether the primary system re-starts transmitting, so a decision will be made to switch the secondary system to another spectrum band or not.

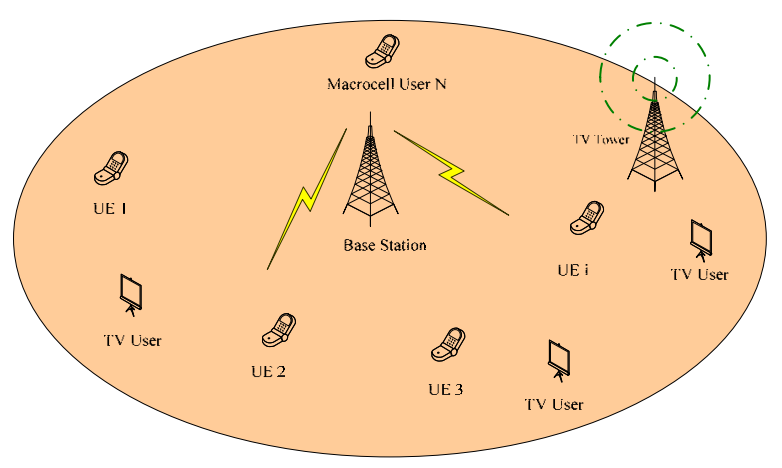

Figure 1. Wireless communication system existing in TV system

In this paper, we focus on the latter scenario in OFDM systems. That is, the OFDM system is working on a spectrum band as a secondary system, and the spectrum sensing is aiming for detect whether the primary system begins transmitting signals again.

\section{Technique Analysis}

\subsection{OFDM System Guard Band}

In OFDM system, system BW is composed by transmission BW and guard BW. Guard BW is used to prevent inter-system interference (interference from power leakage of OFDM system), and there is no signals

\footnotetext{
a Corresponding author: 13709759761@139.com
} 

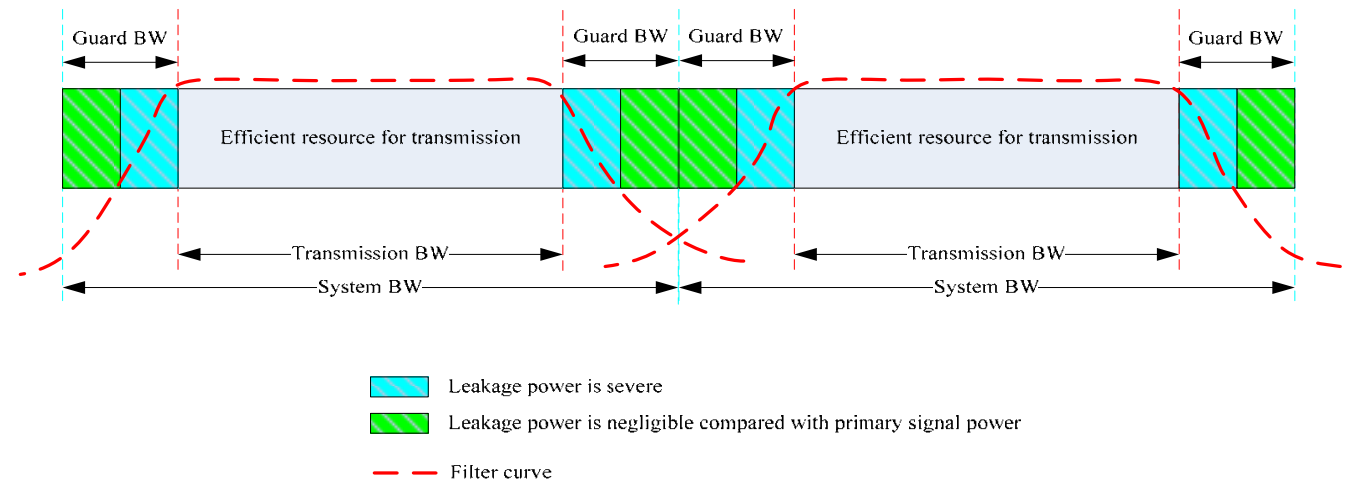

Figure 2. OFDM system guard BW illustration

on the guard BW except for leakage power from neighbor transmission $\mathrm{BW}$ and white noise. Figure 2 gives an illustration of how guard BW prevents the power leakage from inter OFDM systems. From figure 2, it can be considered that the guard $\mathrm{BW}$ is composed by two parts: the inner part and the outer part. We define the outer part of guard BW as leakage-negligible-BW here.

Under the above inner and outer parts based participation, the signals in the inner part of the guard BW will mostly be affected by the signal leakage due to non-ideal filter, while the signals in the outer part of the guard BW will mostly be influenced by the signal leakage due to inter-system interference. However, the leakage power is restricted to a low level so that there is little impact for sensing to decide whether primary signals exist or not. Further, for those users with welldesigned filters, the signal leakage from transmission BW can be reduced to a very low level. As a result, we can effectively use the guard BW, specifically, the named leakage-negligible-BW, to sense the primary system.

Here we take LTE/LTE-A system for example, which is the most promising OFDM technique based cellular wireless communication system. The guard BW configurations of LTE/LTE-A system are given by Table 1.

Table 1. LTE/LTE-A system guard BW configuration

\begin{tabular}{|c|c|c|}
\hline System BW & Transmission BW & Guard BW \\
\hline $20 \mathrm{M}$ & $18 \mathrm{M}$ & $2 \mathrm{M}$ \\
\hline $15 \mathrm{M}$ & $13.5 \mathrm{M}$ & $1.5 \mathrm{M}$ \\
\hline $10 \mathrm{M}$ & $9 \mathrm{M}$ & $1 \mathrm{M}$ \\
\hline $5 \mathrm{M}$ & $4.5 \mathrm{M}$ & $0.5 \mathrm{M}$ \\
\hline $3 \mathrm{M}$ & $2.7 \mathrm{M}$ & $0.3 \mathrm{M}$ \\
\hline $1.4 \mathrm{M}$ & $1.08 \mathrm{M}$ & $0.32 \mathrm{M}$ \\
\hline
\end{tabular}

\subsection{Prior Art}

One of the major problems in spectrum sensing is how to sense primary system for wireless communication systems without too much change on its frame structure or too much signaling complexity. The invention proposed here gives a spectrum sensing method with no frame structure changed and little signaling complexity increased for OFDM systems.
There are some contributions about how to sense the spectrum resource of primary system. The most of these contributions are based on time domain QP (Quiet Period), such as setting some subframes as QP $[10,11]$ for sensing. Contribution [10] gives a general in-band sensing method based on QP, and contribution [2] gives a QP based in-band sensing method for TD-LTE system. However, the QP based sensing methods introduce too many changes on system structure, which is illustrated as follows by taking contribution [11] for example.

The method in [11] includes a functional unit which takes in charge of the selection of Up/Down link configuration as in table 2 . It is assumed here that spectrum sensing just occupies the uplink subframes, because the uplink throughput in TD-LTE networks is relatively small in contrast to the downlink communication throughput. The implement steps are as following:

Firstly, select several possible configuration modes according to the required spectrum sensing time to reach enough sensing performance. For example, if it needs $2 \mathrm{~ms}$ to sensing spectrum to reach detection probability of $99 \%$, mode $0,1,3,6$ are optional.

Then, choose certain mode from the four modes mentioned above depending on up and downlink throughput of network.

And finally, base station broadcasts a sensing instruction in the downlink subframe (0 or 5), UEs (User Equipment) sense the unlicensed bands, and report sensing results in the following uplink subframes.

Table 2. Uplink / Downlink subframe configurations for LTE TDD

\begin{tabular}{|c|c|c|c|c|c|c|c|c|c|c|}
\hline \multirow{2}{*}{$\begin{array}{l}\text { Up/Down } \\
\text { configure }\end{array}$} & \multicolumn{10}{|c|}{ Sub-frame number } \\
\cline { 2 - 12 } & 0 & 1 & 2 & 3 & 4 & 5 & 6 & 7 & 8 & 9 \\
\hline 0 & D & S & U & U & U & D & S & U & U & U \\
\hline 1 & D & S & U & U & D & D & S & U & U & D \\
\hline 2 & D & S & U & D & D & D & S & U & D & D \\
\hline 3 & D & S & U & U & U & D & D & D & D & D \\
\hline 4 & D & S & U & U & D & D & D & D & D & D \\
\hline 5 & D & S & U & D & D & D & D & D & D & D \\
\hline 6 & D & S & U & U & U & D & S & U & U & D \\
\hline
\end{tabular}


As explained above, due to a whole subframe or several consecutive subframes being used as quiet periods, these contributions introduce too many changes on current LTE/LTE-A frame structure such as HARQ (Hybrid Automatic Repeat Request) timing and control signaling transmission, which bring great challenges for current frame structure and might not be attractive in standardization as well as practical implementation.

As explained above, due to a whole subframe or several consecutive subframes being used as quiet periods, these contributions introduce too many changes on current LTE/LTE-A frame structure such as HARQ (Hybrid Automatic Repeat Request) timing and control signaling transmission, which bring great challenges for current frame structure and might not be attractive in standardization as well as practical implementation.

\section{Novel In-band Sensing Method}

\subsection{Overall Introduction}

The prior inventions are based on QP, which affect the frame structure severely. Here we propose a guard BW based spectrum sensing method, by which the impact to OFDM system frame structure could be minimized.

The proposed spectrum sensing procedure is based on leakage-negligible-BW, on which power detection or other detection methods can be implemented. For the method of power detection, the leakage-negligible-BW power is very low when no primary signals exist, else could be relative high. For the other sensing method, a somewhat sensing result can also be derived. The sensing result will be reported to BS (Base Station) via uplink transmission to derive a relative high sensing performance by cooperative sensing.

UE category, which symbolizes the UE capability, is reported to BS by uplink transmission. As the power leakage will affect the sensing result on leakagenegligible-BW, those UEs whose filter is good enough will be chosen to sense. So a parameter which is able to indicate the filter performance should be added in UE category.

On one hand, the sensing result on leakagenegligible-BW has to be reported by uplink transmission; on another hand, the filter parameter added in UE category introduces some changes on system standardization. These two points are our novel in this paper.

\subsection{Implementation}

The procedure of proposed spectrum sensing method is:

Step 1: Adding a parameter in UE category to indicate the UE filter capability and reporting to BS;

Step 2: BS selects UEs whose filters are good enough for sensing, and notices these UEs to feed back the sensing results periodically based on leakage-negligibleBW;

Step 3: The selected UEs calculate and report the sensing results to BS periodically;
Step 4: A decision will be made by BS whether to continue reusing the spectrum of primary system or trigger a finer sensing procedure based on these reports.

\subsection{Advantages and Disadvantages}

For our proposed scheme, the spectrum sensing performance will be affected by the UE filter property. In order to avoid sensing performance degradation, we choose those UEs with good filter property by including filter capabilities in UE category to sense. Although the filter property has impact to spectrum sensing, it's not a critical issue. In addition, to avoid sensing performance degradation, a frequency margin can be reserved by the edge of leakage-negligible-BW.

As the guard band always exists for both downlink and uplink, the samples for sensing are time domain continuous, which guarantee the sensing performance. In addition, the proposed method has little impact to system frame structure and little signaling increased, so it's very convenient in practical implementation.

\section{Conclusion}

A novel in-band spectrum sensing method based on guard frequency bandwidth for cognitive OFDM systems is proposed in this paper. The proposed spectrum sensing procedure is based on leakage-negligible-BW, on which power detection or other detection methods can be implemented. The advantage of this in-band sensing method is the impact to OFDM system frame structure could be minimized. So the proposed method is a practical implementation which can be used in cognitive OFDM system.

\section{Acknowledgment}

This work was supported by the International Science \& Technology Cooperation Project of Qinghai (2014HZ-821) and Application Basic Research Project of Qinghai(2015-ZJ-721).

\section{References}

1. H. Simon. Cognitive radio: brain-empowered wireless communications. IEEE Journal on Selected Areas in Communications, 23(2005)

2. J. Mitola. Cognitive radio: making software radios more personal. IEEE Personal Communications, 6(1999)

3. T. Yucek, H. Arslan. A survey of spectrum sensing algorithms for cognitive radio applications. IEEE Communications Surveys \& Tutorials, 11(2009)

4. J. Mitola. Cognitive radio for flexible mobile multimedia communications. Proc. IEEE Int. Workshop MoMuC, (1999)

5. H. Urkowitz. Energy detection of unknown deterministic signals. Proc. IEEE, 55(1967)

6. F. Digham, M. Alouini and M. Simon. On the energy detection of unknown signals over fading channels. IEEE Trans. Commun, 55 (2007) 
7. T. Yucek and H. Arslan. A survey of spectrum sensing algorithms for cognitive radio applications. IEEE Commun. Surveys Tuts, 11( 2009)

8. B. Wang and K. Liu. Advances in cognitive radio networks: A survey. IEEE J. Sel. Topics Signal Process, 5( 2011)

9. E. Axell, G. Leus, E. G. Larsson and H. V. Poor. Spectrum sensing for cognitive radio: State-of-theart and recent advances. IEEE Signal Process. Mag, 29( 2012)

10. IEEE P802.22тм/D0.1, Draft Standard for Wireless Regional Area Networks Part 22: Cognitive Wireless RAN Medium Access Control (MAC) and Physical Layer (PHY) specifications: Policies and procedures for operation in the TV Bands.

11. Z. Feng, P. Zhang, et al. Cooperative cognitive technique based on LTE system. Chinese patent number:201010248004. 